Check for updates

The BMJ

Cite this as: BMJ 2021;375:n3011 http://dx.doi.org/10.1136/bmj.n3011 Published: 03 December 2021

\title{
Covid-19: Antibody boost after third dose varies greatly by vaccine, study finds
}

\section{Elisabeth Mahase}

Six different covid-19 vaccines are safe and effective to use as booster doses in people who previously received the Oxford AstraZeneca or Pfizer BioNTech vaccines, but the antibody boost they provide varies substantially, a study has found. ${ }^{1}$

The Cov-Boost trial looked at the safety, immune response, and side effects of seven vaccines when used as a third vaccine dose, often referred to as a booster dose-AstraZeneca, Curevac, Johnson \& Johnson (Janssen), Moderna, Novavax, Pfizer, and Valneva, while a meningococcal conjugate vaccine was used as a control.

The trial involved 2878 healthy participants over 30 years old who were split across 13 experimental and control arms-seven vaccines plus three at half dose and three control arms.

The study, published in the Lancet, found that the Moderna vaccine produced the biggest antibody response and a half dose of Valneva the smallest.

Among those primed with two doses of AstraZeneca, antibody levels were 32 higher after the Moderna booster than control (24.8 to 42.0) compared with nearly two times higher after a half dose of the Valneva (1.8, 99\% confidence interval 1.5 to 2.3).

For people primed with the Pfizer vaccine, a Moderna booster increased antibody levels 11.5 (9.4 to 14.1) times higher than control while a half dose of Valneva increased levels by 1.3 times ( $99 \%$ CI 1.0 to 1.5$)$. The results were similar for all ages.

The researchers said that the results should be interpreted with caution as they relate to immunogenicity rather than protection against disease.

Trial lead Saul Faust, NIHR clinical research facility director at University Hospital Southampton NHS Foundation Trust, said, "It's encouraging that a wide range of vaccines, using different technologies, show benefits as a third dose to either AstraZeneca or Pfizer. That gives confidence and flexibility in developing booster programmes here in the UK and globally, with other factors like supply chain and logistics also in play."

Reactions to all seven vaccines were similar, with fatigue, headache, and injection site pain most often reported. There were 24 severe adverse events recorded-five in the control group, two in Johnson \& Johnson, five in Valneva, one in half Valneva, one in Pfizer, two in half Pfizer, two in AstraZeneca, one in Curevac, two in Novavax, two in half Novavax, and one in Moderna.

\section{T cell responses}

Looking at $\mathrm{T}$ cell responses, the researchers found that all the study vaccines except AstraZeneca, Valneva, and half dose Valneva "significantly induced cellular responses" in those primed with the AstraZeneca vaccine. For those primed with the Pfizer vaccine, however, all the vaccines produced a significant $\mathrm{T}$ cell response except for a half dose Novavax.

Notably, the researchers found that $\mathrm{T}$ cell responses against the delta and $\beta$ variants were similar to those against the wildtype virus or original Wuhan virus.

Speaking at the Science Media Centre briefing on 1 December, Faust said, "The T cell data, even though we don't properly understand its relation to long term immunity, is showing us that it does seem to have a broader response against all the variant strains, which gives us hope that a variant strain of the virus might be able to be handled, certainly for hospital admissions and death, if not prevention of infection by the current vaccines."

\section{Half doses}

Faust also raised the question of using half instead of full doses of the mRNA vaccines as boosters, as a way to vaccinate more people with fewer resources while still achieving high immune responses.

He said, "We might be able to reduce the doses of the mRNA vaccines when they're used as a third dose in order to increase global vaccine supply. One thing we need are studies of lower doses that can achieve just as high antibody levels and just as good cellular responses but enable us to immunise maybe two, three, or four times the number of people with the same vaccine dose."

The study, funded by the UK Vaccine Taskforce and National Institute for Health Research, had several limitations, including that the interval between second and third doses was shorter in some participants than between their first two doses, meaning that the boost in immunity could be lower than if longer intervals had been used. As only people over 30 were recruited, the researchers said the generalisability of the findings to younger age groups is limited.

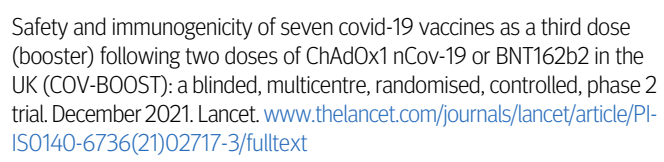
(booster) following two doses of ChAdOx1 nCov-19 or BNT162b2 in the UK (COV-BOOST): a blinded, multicentre, randomised, controlled, phase 2 trial. December 2021. Lancet. www.thelancet.com/journals/lancet/article/PI IS0140-6736(21)02717-3/fulltext

This article is made freely available for use in accordance with BMJ's website terms and conditions for the duration of the covid-19 pandemic or until otherwise determined by BMJ. You may use, download and print the article for any lawful, 
non-commercial purpose (including text and data mining) provided that all copyright notices and trade marks are retained. 